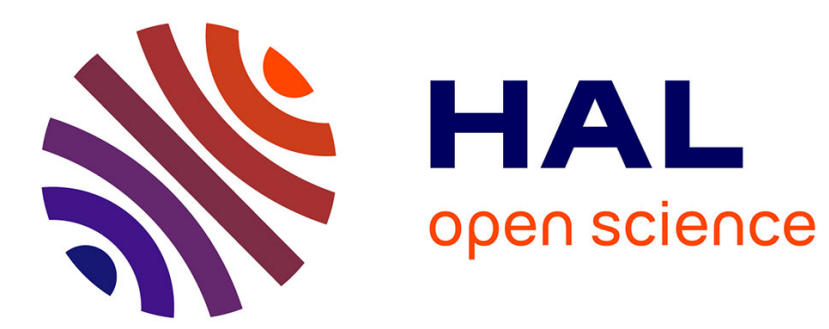

\title{
Les lecteurs parlent
}

Michel Cointat

\section{- To cite this version:}

Michel Cointat. Les lecteurs parlent. 1965, pp.42. 10.4267/2042/24686 . hal-03535219

\section{HAL Id: hal-03535219 \\ https://hal.science/hal-03535219}

Submitted on 19 Jan 2022

HAL is a multi-disciplinary open access archive for the deposit and dissemination of scientific research documents, whether they are published or not. The documents may come from teaching and research institutions in France or abroad, or from public or private research centers.
L'archive ouverte pluridisciplinaire HAL, est destinée au dépôt et à la diffusion de documents scientifiques de niveau recherche, publiés ou non, émanant des établissements d'enseignement et de recherche français ou étrangers, des laboratoires publics ou privés. 


\section{LES LECTEURS PARLENT}

\section{A propos de la forêt de Mazan}

Nous recevons d'un de nos lecteurs, M. M. Cointat, Directeur Général de la Production et des Marchés au ministère de l'Agriculture, la lettre suivante:

"Je ne saurais trop féliciter M. le Chef de District GaIllard pour son excellente étude sur l'historique de la forêt de Mazan (R.F.F. $\mathrm{n}^{\circ} 11$, novembre 1964).

Forestier égaré dans le maquis des soucis de l'Agriculture, j'ai pris beaucoup de plaisir à lire le destin mouvementé de ce beau massif de l'Ardèche et dont la vieillesse majestueuse eut encore dans un passé récent à subir l'avertissement d'une nature capricieuse certes! mais soucieuse d'équilibre et d'harmonie.

Cette lecture, particulièrement intéressante, me permet de faire quelques réflexions personnelles qui sont susceptibles d'intéresser les fidèles de la Revue Forestière Française.

I1 y aurait de multiples conclusions à tirer des documents et des faits relevés de M. GaILlaRD: « Moulins à scie, droits d'usage, charbonnières, etc... 》

Je ne retiendrai tout d'abord qu'une petite critique qui n'affecte en rien la valeur de l'étude mais qui intéresse un aspect de la gestion des forêts ecclésiastiques. Le livre de la fondation de Mazan précise que le vénérable Amédée commença par « cultiver les terres incultes, à raser les forêts, à extirper les ronces et à planter des arbres d'espèces variées 》.

Je ne crois pas comme le pense M. GaILLARD, que le sapin faisait partie de ces « arbres d'espèces variées ». Lorsque les moines n'étaient pas essentiellement agriculteurs et défricheurs comme les bénédictins, ils étaient " éleveurs 》 spécialistes du pâturage, qu'il s'agisse de «bêtes aumailles 》 ou de «bêtes menues 》.

Ainsi, les cisterciens ou les chartreux étaient plus près de la forêt qui leur apportait une ambiance de recueillement, mais qui fournissait aussi un terrain de parcours extraordinaire pour le bétail plutôt constitué de porcs que de bovins.

Souvent on a dit que ces ordres étaient respectueux de la forêt et grâce à cet amour pour la sylve primitive, ont permis de conserver de nombreux massifs. Sans mésestimer l'action favorable des moines pasteurs et forestiers, l'honnêteté conduit à dire qu'ils ont entretenu la forêt dans un but plus matérialiste qu'idéaliste, plus temporel que spirituel, car comme tout le monde, les monastères avaient besoin de revenus substantiels pour vivre.

Je reste donc persuadé que ces « arbres d'espèces variées 》 remplaçant des peuplements naturels plus ou moins dégradés avaient pour rôle d'améliorer 
le pâturage et l'alimentation du bétail, puisque comme le note le Chef de District GaIllard, il est difficile de concevoir des plantations d'arbres fruitiers (au sens actuel de cette expression!) à une altitude de $1135 \mathrm{~m}$.

Mon sentiment va ainsi plutôt au hêtre qui donne des faînes appréciées par les animaux. Il n'est pas non plus invraisemblable de songer au chêne et à ses glands malgré un climat très rigoureux qui ne lui convient guère.

J'ai cru bien faire, il y a déjà longtemps, d'écrire dans la R.F.F. que probablement pour des raisons analogues, les moines chartreux de Valbonne, près de Pont-Saint-Esprit dans le département du Gard, avaient planté du hêtre en plein pays méditerranéen bien que là aussi le climat ne convenait guère à cette essence. Pour des raisons diverses, le hêtre s'est maintenu jusqu'à nos jours dans cette forêt, et j'ai reçu à ce sujet d'amicales remontrances de la part de ceux qui considéraient le hêtre de Valbonne comme une relique glaciaire. Aujourd'hui la forêt de Mazan m'invite à persister dans mon idée à croire au bon sens pratique de nos excellents moines, qu'ils soient de 1'Ardèche ou du Gard.

Mais venons-en à une réflexion plus importante qui a trait à la tragique 《catastrophe 》de 1957. Je suis reconnaissant à M. le Chef de District GAILlARD de nous avonr fait vivre avec intensité cette nuit de « grande peur 》, triste cadeau de Noël pour ces braves gens enracinés dans cette pittoresque région. Cette nuit-là, il était possible de mesurer combien, malgré l'intelligence humaine, est faible la force de l'homme, face aux forces de la nature. Et les peuples oublient trop souvent que pour satisfaire l'équilibre biologique, il est indispensable d'opposer une force naturelle à une autre force naturelle.

Je crois en la vertu de la sagesse paysanne et qui mieux que les forestiers locaux que peut traduire cette sagesse et sentir les erreurs du passé.

Je relève dans cet article sur la forêt de Mazan, quelques phrases caractéristiques qui expriment les inquiétudes du responsable d'un domaine:

«Les erreurs qui se succédèrent, erreurs découlant sans doute d'un esprit de conservation trop poussé, firent que la forêt domaniale de Mazan devint en moins d'un siècle une forêt vieillie... 》

«Elle est enfin pour le forestier qui la connait plus que tout autre et qui l'aime, une trop vieille forêt, ou plus exactement, une « forêt vieillie 》 qu'il faut à tout prix rajeunir...»

Mais immédiatement l'enthousiasme sympathiquement chauvin du forestier, confiant dans la puissance de la vie, reprend le dessus:

"La forêt de Mazan quoique très éprouvée, reste malgré cela dans l'esprit du public « la plus belle forêt de l'Ardèche ».

«Tout compte fait, la « catastrophe 》n'aurait-elle pas contribué pour une grande part à ce rajeunissement? 》

« Aurait-on envisagé aussi vite, sans cela, les opérations destinées à éliminer les trop vieux bois, à provoquer la régénération naturelle et à imposer s'il le faut, la régénération artificielle... 》

Et voilà où je voulais en venir!

Pendant des années et des années, on a accumulé des milliers de mètres cubes, pour des motifs conservatoires qui étaient certainement justifiés à l'origine, on a laissé vieillir doucement les peuplements et tout cela en définitive pour rien. L'économie n'y a sûrement guère gagné, et la forêt, à la suite d'une saute d'humeur du Destin, qui semble bien être un sévère rappel à l'ordre, se retrouve meurtrie et dégradée.

Accumuler du « cube » n'est pas forcément améliorer un peuplement, ne conduit pas fatalement les massifs vers des stades plus « progressés 》 vers des formes meilleures.

Un vigoureux semis en brosse de chêne, de hêtre ou de sapin constitue une beauté forestière bien plus grande que ces futaies « cathédrales majestueuses mais dépérissantes qui enchantent le promeneur, qui offrent une beauté touristique indéniable, mais qu'il faudra bien remplacer un jour, car la vieillesse n'est pas éternelle 》. 
Un équilibre harmonieux ne peut être réalisé dans ce monde complexe qu'est la forêt, où le lent et puissant boưillonnement de la vie crée de perpétuels mouvements, d'éternelles progressions, que si la santé des peuplements rayonne, que si les arbres, qui composent le squelette de cet univers, sont vigoureux, élancés, ivres de soleil et de pluie et « fous d'oiseaux 》 pour reprendre l'expression d'Eluard.

Combien de massifs se trouvent en France dans la situation de la forêt de Mazan, qui n'offre sans doute aucune originalité à ce sujet.

Est-ce que l'économie de notre pays n'aurait pas gagné à une conception plus dynamique, à une action plus réaliste? Est-ce que dans le même temps, la forêt n'y aurait pas gagné en puissance, en santé, en équilibre?

Le maintien d'une politique essentiellement conservatoire, créée à juste titre à une époque différente de la nôtre, ressemble étrangement à ce protectionnisme constitué à la fin $\mathrm{du} \mathrm{XIX}^{\mathrm{\theta}}$ siècle par le Ministre Jules Méline dans le domaine agricole, protectionnisme aujourd'hui outrancier et contre lequel nous sommes obligés d'opposer une politique plus libérale et mieux adaptée aux conditions économiques nouvelles.

Et pourtant Jules Méline, dans sa clairvoyance, avait dit du haut de la tribune du Sénat, que ce protectionnisme ne devait être que transitoire; qu'il devait permettre à l'agriculture de s'adapter, de se moderniser, et ensuite que progressivement, il devrait s'effacer au fur et à mesure que l'économie agricole deviendrait compétitive.

Mais l'agriculture française s'est confortablement installée à l'abri de cette protection, on a oublié les sages paroles de Méline et maintenant au moment où le marché commun européen entr'ouvre sa porte, il faut rattraper le temps perdu au prix d'un effort colossal.

Je crains qu'il n'en soit de même pour la forêt.

Certains ont voulu opposer les économistes aux contemplatifs. Pour ceux qui connaissent le langage de la forêt et qui aiment ce domaine d'ombre, de fraîcheur et de calme, il ne peut y avoir une telle opposition. Et je voudrais profiter de ce propos, pour répondre à $M$. le Général VIDAL et à M. Rivaillon, qui ces derniers mois, et dans cette Revue, ont amicalement correspondu au sujet de «l'amour de la forêt... et du métier de forestier 》.

La forêt garde une double mission: protéger l'homme contre la nature et contre lui-même, apporter à l'homme le bois dont il a besoin.

Certains massifs ont un rôle de protection, d'autres un rôle d'espaces verts, de « poumons》 pour 《 citadins épuisés 》. Il convient de les traiter comme tels.

Mais aussi d'autres peuplements ont une mission « d'usines à bois 》. Ils doivent produire du bois, mais pas n'importe quel bois. Ils doivent produire ce qui convient au marché, ce qui répond aux désirs et aux besoins de l'économie. Dans cette optique, la forêt doit être adaptée en permanence et ceci d'autant plus impérativement que la forêt évolue lentement alors que les fluctuations économiques sont souvent brusques et rapides.

I1 est indispensable dans ces forêts de respecter l'équilibre naturel pour ne pas gaspiller le capital biologique, mais la rentabilité, la productivité, les investissements économiques y prennent une place de choix.

Je voulais profiter de l'étude de M. le Chef de District Gaillard sur la forêt de Mazan pour essayer de montrer qu'un économiste hybridé de forestier aurait probablement répondu d'une façon plus satisfaisante tant aux nécessités du marché qu'aux impératifs naturels.

Je serais heureux si ces quelques réflexions pouvaient prendre place dans la rubrique de la Revue «Les lecteurs parlent... ».

M. Cointat. 